\title{
Dinámica del recambio de lípidos y sus implicancias en la obesidad durante el ciclo vital
}

\section{SR. EDITOR}

La obesidad constituye un factor de riesgo para múltiples enfermedades crónicas no transmisibles como diabetes mellitus tipo 2, síndrome metabólico, enfermedades cardiovasculares y cáncer'. Por lo tanto, para mejorar el manejo de la obesidad y sus co-morbilidades, es clave conocer mejor como ocurre la dinámica lipídica, es decir, la regulación del almacenamiento y remoción de triacilglicéridos (TAGs) en el tejido adiposo.

Un reciente artículo de Arner et $\mathrm{al}^{2}$, publicado en Nature Medicine, realizó un aporte fundamental para la comprensión de la dinámica del tejido adiposo a través del ciclo vital. En una prueba controlada aleatorizada se midieron las tasas de almacenamiento y remoción de lípidos del tejido adiposo subcutáneo en 56 sujetos en un periodo de 13 a 16 años. Para estudiar la dinámica lipídica, los investigadores utilizaron una interesante estrategia que consistió en medir el contenido de carbono $14\left({ }^{14} \mathrm{C}\right)$ que es naturalmente incorporado en los TAGs a partir del consumo de nutrientes orgánicos, los cuales en último término provienen de la fijación del $\mathrm{CO}_{2}$ atmosférico realizada mediante fotosíntesis por los vegetales. Debido a lo anterior, los niveles de ${ }^{14} \mathrm{C}$ de los TAGs varían en paralelo a los cambios de concentración de ${ }^{14} \mathrm{C}$ atmosférico. Los niveles de ${ }^{14} \mathrm{C}$ en la atmósfera aumentaron sustancialmente debido a los ensayos nucleares durante la guerra fría, pero con posterioridad al tratado de prohibición parcial de ensayos nucleares en 1963, los niveles de ${ }^{14} \mathrm{C}$ han disminuido exponencialmente debido a la difusión del ${ }^{14} \mathrm{C}$ desde la atmósfera a los océanos o el espacio exterior. Por lo tanto, una mayor cantidad de ${ }^{14} \mathrm{C}$ en lípidos subcutáneos, implicaría que estos fueron almacenados más tempranamente en el tejido adiposo ya que fueron capturados de los alimentos cuando había mayores niveles de ${ }^{14} \mathrm{C}$ en el ambiente. De esta manera, los niveles de ${ }^{14} \mathrm{C}$ constituyen una medida de la antigüedad de los lípidos en nuestros tejidos, y a través de ella fue posible determinar la tasa de almacenamiento (o formación) de lípidos y de remoción (o salida) de lípidos del tejido adiposo en el estudio publicado por Arner et al².

La investigación encontró que el envejecimiento está asociado a una disminución de la capacidad para
Marcelo Villagrán ${ }^{1 *}$, Claudia Troncoso-Pantoja ${ }^{2}$, Ana María Leiva ${ }^{3}$, María Adela Martínez-Sanguinetti ${ }^{4}$, Fanny Petermann-Rocha ${ }^{5,6}$, Carlos Celis-Morales ${ }^{5}$.

1. Departamento de Ciencias Básicas. Universidad Católica de la Santísima Concepción. Concepción. Chile. 2. Departamento de Salud Pública, CIEDE-UCSC, Facultad de Medicina, Universidad Católica de la Santísima Concepción, Concepción, Chile. 3. Instituto de Anatomía, Histología y Patología, Facultad de Medicina, Universidad Austral de Chile, Valdivia, Chile. 4. Instituto de Farmacia, Facultad de Ciencias, Universidad Austral de Chile, Valdivia, Chile. 5. BHF Glasgow Cardiovascular Research Centre, Institute of Cardiovascular and Medical Sciences, University of Glasgow, Glasgow, United Kingdom.

${ }^{6}$ Institute of Health and Wellbeing, University of Glasgow, Glasgow, United Kingdom.

*Dirigir correspondencia a: Marcelo Villagrán. Departamento de Ciencias Básicas Facultad de Medicina. Universidad Católica de la Santísima Concepción. Alonso de Ribera 2850. Concepción. Chile. Teléfono: 412345814. Email: marcelo.villagran@ucsc.cl

Este trabajo fue recibido el 06 de noviembre de 2019. Aceptado con modificaciones: 20 de enero de 2020 . Aceptado para ser publicado: 29 de marzo de 2020.

remover los lípidos del tejido adiposo, observándose que la permanencia de los lípidos en el tejido adiposo aumentó significativamente en $0,6 \pm 0,8$ años en los 13 años de seguimiento. Dado que la utilización de las reservas de TAGs requiere de la movilización de lípidos desde el tejido adiposo, se estudió la correlación entre la capacidad de pérdida de peso corporal con la tasa de remoción de lípidos; sin embargo, contrariamente a lo esperado, no se encontró tal correlación. Estos datos implican que la pérdida de peso corporal a edades más avanzadas depende fundamentalmente de la disminución del almacenamiento de lípidos ya que la tasa de remoción de lípidos disminuye a medida que envejecemos. De hecho, los individuos que mantuvieron estable su tasa de almacenamiento de lípidos 
fueron ganando progresivamente un $20 \%$ de peso corporal en el curso de 13 años de seguimiento ${ }^{2}$. Esto implica que, para mantener un peso constante, es necesario disminuir la ingesta energética a medida que se avanza en edad.

A pesar de que en la cohorte estudiada los cambios de peso corporal fueron bajos, se pudo observar un aumento de peso corporal en personas que no disminuyeron su tasa de almacenamiento de lípidos a medida que envejecían. Para corroborar los hallazgos anteriores, los investigadores recurrieron a una cohorte de sujetos sometidos a cirugía bariátrica, en quienes se registró una disminución de $26 \%$ en el índice de masa corporal (IMC) durante cinco años de seguimiento. En concordancia con la cohorte anterior, la gran pérdida de peso corporal se correlacionó con una disminución en la tasa de almacenamiento de lípidos y no con una mayor tasa de remoción de lípidos. Otro hallazgo interesante en esta última cohorte fue que aquellos individuos con menor tasa de remoción de lípidos fueron los que presentaron la mejor capacidad para mantener la pérdida de peso en el largo plazo ${ }^{2}$. Una baja tasa de remoción de lípidos previa a la marcada pérdida de peso permitió que estos sujetos tuviesen un margen para aumentar su tasa de remoción de lípidos y de esta manera adaptarse a los cambios metabólicos impuestos por la pérdida de peso corporal.

Los resultados del estudio de Arner et $\mathrm{al}^{2}$ están de acuerdo con la evidencia previa que describe una ganancia de tejido adiposo visceral y aumento de IMC durante el envejecimiento ${ }^{3}$. El grupo de los adultos mayores no ha sido ajeno a la epidemia global de obesidad, observando que el $76,8 \%$ de los mayores de 65 años padecen sobrepeso u obesidad en Chile ${ }^{4}$. La obesidad en las personas mayores constituye un problema difícil de abordar ya que existe ambigüedad para distinguir entre la ganancia natural de tejido adiposo durante el envejecimiento y la obesidad propiamente tal. Además de la ganancia fisiológica de tejido graso, los adultos mayores experimentan una pérdida progresiva de tejido muscular, de manera que los criterios de definición obesidad basados en el IMC no resultan adecuados para ellos 5 . Por otro lado, existe controversia sobre cómo abordar la obesidad en las personas mayores debido a la evidencia que indica que el sobrepeso moderado sería un factor protector de la mortalidad en este grupo etario, lo cual se conoce como la paradoja de la obesidad ${ }^{6}$. Ante los desafíos de una creciente obesidad y el aumento progresivo la población de personas mayores, los datos aportados por el estudio de Arner et $\mathrm{al}^{2}$ contribuyen a aclarar aspectos regulatorios de la dinámica lipídica durante el envejecimiento, evidenciando la necesidad de realizar un control acucioso de la alimentación en los adultos mayores para el control del sobrepeso, así como también, fomentar la práctica de actividad física orientada a mitigar la disminución natural de masa muscular y de capacidad aeróbica asociadas a esta etapa del ciclo vital.

\section{BIBLIOGRAFÍA}

1. Gadde KM, Martin CK, Berthoud HR, Heymsfield SB. Obesity: Pathophysiology and Management. I Am Coll Cardiol 2018; 71: 69-84.

2. Arner P, Bernard S, Appelsved L, Fu KY, Andersson DP, Salehpour $M$, et al. Adipose lipid turnover and long-term changes in body weight. Nat Med 2019; 25: 1385-1389.

3. Kuk LL, Saunders TJ, Davidson LE, Ross R. Age-related changes in total and regional fat distribution. Ageing Res Rev 2009; 8: 339-348.

4. MINSAL. Subsecretaria de Salud Pública, División de Planificación Sanitaria, Departamento de Epidemiología. 2017. Encuesta Nacional de Salud 2016-2017. Chile.

5. Batsis JA, Villareal DT. Sarcopenic obesity in older adults: aetiology, epidemiology and treatment strategies. Nat Rev Endocrinol 2018; 14: 513-537.

6. Elagizi A, Kachur S, Lavie Cl, Carbone S, Pandey A, Ortega FB, et al. An Overview and Update on Obesity and the Obesity Paradox in Cardiovascular Diseases. Prog Cardiovasc Dis 2018; 61: 142-150. 